\title{
Implementation of the time-dependent configuration-interaction singles method for atomic strong-field processes
}

\author{
Loren Greenman, ${ }^{1}$ Phay J. Ho, ${ }^{2}$ Stefan Pabst,,${ }^{2,3,}{ }^{*}$ Eugene Kamarchik, ${ }^{1}$ David A. Mazziotti, ${ }^{1, \dagger}$ and Robin Santra ${ }^{2,4, \dagger}$ \\ ${ }^{1}$ Department of Chemistry and The James Franck Institute, The University of Chicago, Chicago, Illinois 60637, USA \\ ${ }^{2}$ Argonne National Laboratory, Argonne, Illinois 60439, USA \\ ${ }^{3}$ Institut für Theoretische Physik, Universität Erlangen-Nürnberg, D-91058 Erlangen,Germany \\ ${ }^{4}$ Department of Physics, The University of Chicago, Chicago, Illinois 60637, USA
}

(Received 8 June 2010; published 4 August 2010)

\begin{abstract}
We present an implementation of the time-dependent configuration-interaction singles (TDCIS) method for treating atomic strong-field processes. In order to absorb the photoelectron wave packet when it reaches the end of the spatial grid, we add to the exact nonrelativistic many-electron Hamiltonian a radial complex absorbing potential (CAP). We determine the orbitals for the TDCIS calculation by diagonalizing the sum of the Fock operator and the CAP using a flexible pseudospectral grid for the radial degree of freedom and spherical harmonics for the angular degrees of freedom. The CAP is chosen such that the occupied orbitals in the Hartree-Fock ground state remain unaffected. Within TDCIS, the many-electron wave packet is expanded in terms of the Hartree-Fock ground state and its single excitations. The virtual orbitals satisfy nonstandard orthogonality relations, which must be taken into consideration in the calculation of the dipole and Coulomb matrix elements required for the TDCIS equations of motion. We employ a stable propagation scheme derived by second-order finite differencing of the TDCIS equations of motion in the interaction picture and subsequent transformation to the Schrödinger picture. Using the TDCIS wave packet, we calculate the expectation value of the dipole acceleration and the reduced density matrix of the residual ion. The technique implemented will allow one to study electronic channel-coupling effects in strong-field processes.
\end{abstract}

DOI: 10.1103/PhysRevA.82.023406

PACS number(s): 32.80.Rm, 31.15.A-, 02.70.-c

\section{INTRODUCTION}

With the invention of the technique of chirped pulse amplification (CPA), modern high-power laser systems can now easily produce high repetition-rate femtosecond laser pulses, leading to peak optical intensities greater than $10^{14} \mathrm{~W} / \mathrm{cm}^{2}$ or as high as $10^{20} \mathrm{~W} / \mathrm{cm}^{2}$ in the focal region. The availability of such intense radiation has opened up new and important venues of research in atomic, molecular, and optical (AMO) physics. High photon-flux radiation can drive atomic and molecular systems into nonlinear regimes and initiate nonperturbative single-atom and single-molecule intense-field phenomena, including multiphoton ionization [1-17], above-threshold ionization (ATI) [18-27], high-harmonic generation (HHG) [28-42], and nonsequential multielectron ionization [43-52].

Understanding these field-induced phenomena has tremendous technological implications. HHG, for example, has been used as a convenient tool for the production of coherent XUV and soft x-ray radiation [33-37] and attosecond pulses [53,54]. These novel light sources have enabled ultrafast molecular probing [55-72] and the study of AMO physics in the attosecond regime [53,54,73-77]. As a result, various theoretical methods have been developed to investigate strong-field AMO physics. Among these methods, solving the time-dependent Schrödinger equation provides

\footnotetext{
*Present address: Center for Free-Electron Laser Science, DESY and University of Hamburg, Germany.

†damazz@uchicago.edu

${ }^{\ddagger}$ Corresponding author: robin.santra@cfel.de; present address: Center for Free-Electron Laser Science, DESY and University of Hamburg, Germany.
}

the most accurate description. Unfortunately, this method is practically unfeasible for many-electron atoms and molecules beyond helium. Hence, many efforts have been devoted to one-electron formulations of the strong-field problems.

These one-electron formulations are guided by the first step in all the strong-field processes, in which an electron is pulled away from the parent ion by the laser field. Treating this electron as the only active electron has been shown to be sufficient for accurate prediction of single-ionization production [7,78-80], cutoff in the HHG spectrum $[31,80]$, and ATI photoelectron spectra [79] in different atoms. In spite of its successes, this single-active-electron (SAE) approach has its limitations. First, it cannot account for multielectron effects in strong-field processes. For instance, Gordon et al. have shown that the accurate inclusion of multielectron effects is necessary to explain the scaling of the HHG radiation intensity with the atomic number [39]. Second, the SAE theory lacks the dynamical description of the residual ion by focusing only on the wave-packet dynamics of the excited electron. In particular, the theory offers only a single-channel description, in which the ion created is treated to be in a single ionic eigenstate.

Recent experimental efforts have now begun to require an accurate strong-field description of the residual ion. It was revealed from the ATI photo-electron spectrum that $\mathrm{Xe}^{+}$ ions in both the $5 p_{3 / 2}^{-1}$ ground-state and the $5 p_{1 / 2}^{-1}$ excited manifolds were generated in an intense optical pulse [81,82]. Also, experiments at the Advanced Photon Source (APS) using resonant $\mathrm{x}$-ray absorption techniques have unveiled the alignment dynamics of the residual $\mathrm{Kr}^{+}$ions created in a strong optical field $[83,84]$. Furthermore, complete quantum ion state populations, which are the diagonal entries of the ion density matrix in the ion eigenstates basis, were measured experimentally and confirmed theoretically $[85,86]$. 
An approximate one-electron approach that goes beyond the SAE treatment is time-dependent configuration interaction with single excitations (TDCIS). This is an ab initio electronicstructure technique, where the time-dependent wave function is restricted to spin-singlet conserving single-particle excitations from the ground-state determinant. Rohringer and coworkers have shown that TDCIS can be formulated as an effective one-electron theory with coupled channels [87]. This method includes the description of the electronic structure of many-electron atoms and molecules through the Fock operator and allows the determination of the ion density matrix, both the diagonal and off-diagonal elements. Knowledge of the off-diagonal matrix elements reveals the coherence properties associated with the electronic wave-packet dynamics of the residual ion $[85,86,88,89]$. Using a one-dimensional helium model in a strong laser field, it was shown that TDCIS is superior to the SAE approach [87]. There are other rigorous effective one-electron approaches, including the single-configuration and multi-configuration time-dependent Hartree-Fock methods [78,90-92] and time-dependent density-functional theory in the Kohn-Sham formulation $[93,94]$. These methods have known limitations and challenges [95-99]. Recently, Spanner and Patchkovskii used a set of coupled time-dependent single-particle Schrödinger equations derived from a multielectron wave-function ansatz to examine multielectron effects in strong-field one-electron ionization processes [100].

The purpose of this paper is to describe a full numerical implementation of the three-dimensional TDCIS method with an added radial complex absorbing potential (CAP). A set of complex orbitals for the TDCIS calculation is determined by diagonalizing the sum of the Fock operator and the CAP using a flexible pseudospectral grid for the radial degree of freedom and spherical harmonics for the angular degrees of freedom. We found that this complex-orbital formulation of TDCIS has computational advantages, because it allows stable propagation of the TDCIS wave packet. Atomic units are used throughout.

\section{THEORETICAL BACKGROUND}

In this section, we provide the theoretical background for a complex-orbital formulation of TDCIS. We start in Sec. II A with the general description of an atom in linearly polarized laser fields within the configuration-interaction singles (CIS) model. Then, an expression for calculating expectation values and the derivation of the reduced ion density are presented in Secs. IIC and II D, respectively. In Sec. IIE we discuss the Hartree-Fock equations for closed-shell atoms in the presence of a CAP.

\section{A. TDCIS with a CAP}

Within the CIS model, excitations beyond a particle-hole (1p-1h) excitation $\left|\Phi_{i}^{a}\right\rangle$ with respect to the ground state, which is in our case the Hartree-Fock (HF) ground state $\left|\Phi_{0}\right\rangle$, are not considered. Therefore, the wave packet is written in terms of $\left|\Phi_{0}\right\rangle$ and $\left|\Phi_{i}^{a}\right\rangle$,

$$
\begin{aligned}
& |\Psi(t)\rangle=\alpha_{0}(t)\left|\Phi_{0}\right\rangle+\sum_{i, a} \alpha_{i}^{a}(t)\left|\Phi_{i}^{a}\right\rangle, \\
& \left|\Phi_{i}^{a}\right\rangle=\frac{1}{\sqrt{2}}\left\{\hat{c}_{a+}^{\dagger} \hat{c}_{i+}+\hat{c}_{a-}^{\dagger} \hat{c}_{i-}\right\}\left|\Phi_{0}\right\rangle,
\end{aligned}
$$

where $i, j, k, l$ symbolize occupied orbitals, $a, b, c, d$ symbolize virtual orbitals, and $p, q, r, s$ stand for occupied or virtual orbitals. The operators $\hat{c}_{p \sigma}^{\dagger}$ and $\hat{c}_{p \sigma}$ create and annihilate electrons, respectively, in the spin orbital $\left|\varphi_{p \sigma}\right\rangle$, which is an eigenstate of the modified Fock operator

$$
\begin{gathered}
\hat{F}_{\mathrm{CAP}}\left|\varphi_{p \sigma}\right\rangle=\varepsilon_{p}\left|\varphi_{p \sigma}\right\rangle, \\
\hat{F}_{\mathrm{CAP}}=\hat{F}-i \eta \hat{W},
\end{gathered}
$$

where $\hat{F}$ is the Fock operator and $-i \eta \hat{W}$ is the CAP.

The full Hamiltonian of our system is

$$
\hat{H}(t)=\hat{F}_{\mathrm{CAP}}+\hat{V}_{C}-\hat{V}_{\mathrm{HF}}-E_{\mathrm{HF}}-\mathcal{E}(t) \hat{z},
$$

where $\hat{V}_{C}$ is the electron-electron Coulomb interaction, $\hat{V}_{\mathrm{HF}}$ is the Hartree-Fock mean-field potential, $E_{\mathrm{HF}}$ is the Hartree-Fock ground-state energy, $\hat{z}$ is the dipole operator, and $\mathcal{E}(t)$ is the electric field component of the strong-field laser pulse.

By projecting the time-dependent Schrödinger equation onto the states $\left|\Phi_{0}\right\rangle$ and $\left|\Phi_{i}^{a}\right\rangle$, the equations of motion of the expansion coefficients $\alpha_{0}(t)$ and $\alpha_{i}^{a}(t)$ are obtained as

$$
\begin{aligned}
& i \dot{\alpha}_{0}(t)=-\sqrt{2} \mathcal{E}(t) \sum_{i, a} \alpha_{i}^{a}(t) z_{(i, a)}, \\
i \dot{\alpha}_{i}^{a}(t)= & \left(\varepsilon_{a}-\varepsilon_{i}\right) \alpha_{i}^{a}(t)+\sum_{i^{\prime}, a^{\prime}} \alpha_{i^{\prime}}^{a^{\prime}}(t)\left(2 v_{\left(a, i^{\prime}, i, a^{\prime}\right)}-v_{\left(a, i^{\prime}, a^{\prime}, i\right)}\right) \\
& -\mathcal{E}(t)\left\{\sqrt{2} \alpha_{0}(t) z_{(a, i)}+\sum_{a^{\prime}} \alpha_{i}^{a^{\prime}}(t) z_{\left(a, a^{\prime}\right)}\right. \\
& \left.-\sum_{i^{\prime}} \alpha_{i^{\prime}}^{a}(t) z_{\left(i^{\prime}, i\right)}\right\}
\end{aligned}
$$

The projection is done with respect to the symmetric inner product discussed in Sec. IIC. The matrix elements in Eqs. (5) are defined via this symmetric inner product [101]:

$$
\begin{gathered}
z_{(p, q)}=\left(\varphi_{p}|\hat{z}| \varphi_{q}\right), \\
v_{(p, q, r, s)}=\left(\varphi_{p} \varphi_{q}\left|1 / \hat{r}_{12}\right| \varphi_{r} \varphi_{s}\right) .
\end{gathered}
$$

We include parentheses in the subscripts of these matrix elements in order to differentiate them from

$$
\begin{gathered}
z_{p, q}=\left\langle\varphi_{p}|\hat{z}| \varphi_{q}\right\rangle, \\
v_{p, q, r, s}=\left\langle\varphi_{p} \varphi_{q}\left|1 / \hat{r}_{12}\right| \varphi_{r} \varphi_{s}\right\rangle,
\end{gathered}
$$

which are defined by the standard Hermitian inner product. Explicit expressions for the matrix elements in Eqs. (5) may be found in the Appendix.

\section{B. Complex absorbing potential}

When the wave packet reaches the end of the numerical grid, artificial reflections arise. These lead to unphysical results, but can be suppressed by applying a complex absorbing potential (CAP) [102-107] near the end of the grid. As a result, the CAP only affects virtual orbitals. Occupied orbitals are localized near the origin and, therefore, are not influenced by the CAP. Additionally, the CAP does not mix occupied with virtual orbitals. The CAP strength $\eta$ has to be chosen carefully to avoid reflections either off the grid wall or off the CAP $[105,107]$. The explicit form of our CAP is

$$
W(r)=h\left(r-r_{\mathrm{abs}}\right)\left(r-r_{\mathrm{abs}}\right)^{2},
$$


where $h(x)$ is the Heaviside step function, and $r$ is the distance from the origin. This CAP is zero until a radius $r_{\text {abs }}$, after which it is a quadratically growing potential.

\section{Expectation values}

Since $\hat{F}_{\mathrm{CAP}}$ is not Hermitian, the orthogonality relations for its eigenstates are not given in terms of the ordinary Hermitian inner product. If $\left|\varphi_{p}\right\rangle \equiv \mid \varphi_{p}$ ) is a right eigenvector of $\hat{F}_{\mathrm{CAP}}$, then $\left\langle\varphi_{p}\right|$ is generally not a left eigenvector. There is, nevertheless, a one-to-one mapping between right and left eigenvectors. The left eigenvector corresponding to $\left|\varphi_{p}\right\rangle$ is denoted $\left(\varphi_{p} \mid\right.$. The corresponding orthogonality relations read

$$
\left(\varphi_{p} \mid \varphi_{q}\right)=\left(\varphi_{p}\left|\varphi_{q}\right\rangle=\delta_{p, q} .\right.
$$

From the orthogonality relations of the orbitals follow the orthogonality relations of the $1 \mathrm{p}-1 \mathrm{~h}$ excitations,

$$
\left(\Phi_{i}^{a} \mid \Phi_{j}^{b}\right)=\left(\Phi_{i}^{a}\left|\Phi_{j}^{b}\right\rangle=\delta_{a, b} \delta_{i, j} .\right.
$$

Since the $\left|\Phi_{i}^{a}\right\rangle$ are not orthogonal with respect to the Hermitian inner product, we define an overlap integral between $1 \mathrm{p}-1 \mathrm{~h}$ excitations as

$$
\left.\left\langle\Phi_{i}^{a} \mid \Phi_{j}^{b}\right\rangle=\left\langle\Phi_{i}^{a}\right| \Phi_{j}^{b}\right)=\delta_{i, j}\left\langle\varphi_{a} \mid \varphi_{b}\right\rangle=\delta_{i, j} o_{a, b} .
$$

Since the complex absorbing potential does not affect the occupied orbitals $\varphi_{i}$, the orthogonality relation between different $\varphi_{i}$ survives in the Hermitian inner product in Eq. (11).

The expectation value of an operator $\hat{A}$ is defined via the Hermitian inner product. By expanding $\langle\Psi(t)|\hat{A}| \Psi(t)\rangle$ using Eq. (1), we obtain

$$
\begin{aligned}
\langle A\rangle= & \left|\alpha_{0}\right|^{2} 2 \sum_{j} A_{j, j}+\sum_{i, a} 2 \sqrt{2} \operatorname{Re}\left(\alpha_{0}^{*} \alpha_{i}^{a} A_{i, a}\right) \\
& +\sum_{i, a, a^{\prime}} \operatorname{Re}\left(\alpha_{i}^{a *} \alpha_{i}^{a^{\prime}} A_{a, a^{\prime}}\right)-\sum_{i, i^{\prime}, a, a^{\prime}} \operatorname{Re}\left(\alpha_{i}^{a *} \alpha_{i^{\prime}}^{a^{\prime}} A_{i^{\prime}, i} o_{a, a^{\prime}}\right) \\
& +\sum_{i, a, a^{\prime}} \operatorname{Re}\left(\alpha_{i}^{a *} \alpha_{i}^{a^{\prime}} o_{a, a^{\prime}} 2 \sum_{j} A_{j, j}\right) .
\end{aligned}
$$

In order to compute the dipole acceleration, which is required for describing high-harmonic generation, it is possible to calculate the expectation value of the dipole moment and then calculate its second time derivative [96]. Alternatively, the expectation value of the dipole acceleration can be calculated directly. To this end, we employ the dipole acceleration operator obtained using the exact atomic Hamiltonian $[39,108]$. In the atomic case, assuming that the laser field is linearly polarized along the $z$ axis, this operator is given by

$$
\hat{a}=\frac{Z \cos \theta}{\hat{r}^{2}},
$$

where $Z$ is the nuclear charge. Since atomic eigenstates are parity eigenstates, it follows that $a_{i, i}=0$. This simplifies the evaluation of Eq. (12) when $\hat{A}=\hat{a}$.

\section{Reduced ion density matrix}

In order to construct the reduced ion density matrix (IDM) from the state $|\Psi(t)\rangle$, the trace over the unobserved photoelectron of the total density matrix $\hat{\tilde{\rho}}(t)$ has to be performed [109]:

$$
\begin{gathered}
\hat{\tilde{\rho}}^{\mathrm{IDM}}(t)=\operatorname{Tr}_{a}[\hat{\tilde{\rho}}(t)], \\
\tilde{\tilde{\rho}}=|\Psi(t)\rangle\langle\Psi(t)|,
\end{gathered}
$$

where $\operatorname{Tr}_{a}$ stands for the trace over all virtual orbitals $a$ [cf. Eq. (16a)] and $\hat{\tilde{\rho}}^{\mathrm{IDM}}(t)$ symbolizes the ion density matrix. Since the virtual orbitals do not obey the Hermitian orthogonality relations [see Eqs. (10) and (11)], we have to be careful to define the trace in Eq. (14a). The explicit form of the density matrix can be derived from the norm $\|\Psi(t)\|^{2}$,

$$
\begin{aligned}
\langle\Psi(t) \mid \Psi(t)\rangle & \left.=\sum_{I}\langle\Psi(t)| \Phi_{I}\right)\left(\Phi_{I}|\Psi(t)\rangle\right. \\
& =\sum_{I}\left(\Phi_{I}|\Psi(t)\rangle\langle\Psi(t)| \Phi_{I}\right)=\operatorname{Tr}[\tilde{\tilde{\rho}}(t)],
\end{aligned}
$$

where $\operatorname{Tr}$ stands for the trace over the entire configuration space $I$, i.e., all $1 \mathrm{p}-1 \mathrm{~h}$ excitations $\Phi_{i}^{a}$ and the $\mathrm{HF}$ ground state $\Phi_{0}$. Based on this, natural definitions of the traces over only occupied and only virtual orbitals in the $1 \mathrm{p}$-1h-configuration space, which are symbolized by $\operatorname{Tr}_{i}$ and $\operatorname{Tr}_{a}$, respectively, are

$$
\begin{aligned}
& \left\{\operatorname{Tr}_{a}[\hat{\tilde{\rho}}(t)]\right\}_{i, j}=\sum_{a}\left(\Phi_{i}^{a}|\Psi(t)\rangle\langle\Psi(t)| \Phi_{j}^{a}\right), \\
& \left\{\operatorname{Tr}_{i}[\hat{\tilde{\rho}}(t)]\right\}_{a, b}=\sum_{i}\left(\Phi_{i}^{a}|\Psi(t)\rangle\langle\Psi(t)| \Phi_{i}^{b}\right) .
\end{aligned}
$$

Hence, the matrix elements of $\hat{\tilde{\rho}}^{\mathrm{IDM}}(t)$ have the form

$\tilde{\rho}_{i, j}^{\mathrm{IDM}}(t)=\sum_{a}\left(\Phi_{i}^{a}|\Psi(t)\rangle\langle\Psi(t)| \Phi_{j}^{a}\right)=\sum_{a, b} \alpha_{i}^{a}(t)\left[\alpha_{j}^{b}(t)\right]^{*} o_{b, a}$,

where we have used the explicit form of the wave function [cf. Eq. (1)]. To analyze the impact of the CAP on the dynamics of $\hat{\tilde{\rho}}(t)$ and $\hat{\tilde{\rho}}^{\mathrm{IDM}}(t)$, it is convenient to go into the interaction picture (labeled $I$ )

$$
\begin{gathered}
\left|\Psi_{I}(t)\right\rangle=e^{i \hat{F} t}|\Psi(t)\rangle, \\
\hat{A}_{I}=e^{i \hat{F} t} \hat{A} e^{-i \hat{F} t},
\end{gathered}
$$

where $\hat{A}$ stands for any operator in the Schrödinger picture. The time evolution of $\left|\Psi_{I}(t)\right\rangle$ is given by

$i \frac{\partial}{\partial t}\left|\Psi_{I}(t)\right\rangle=\left(\hat{V}_{C, I}-\hat{V}_{\mathrm{HF}, I}-E_{\mathrm{HF}}-\mathcal{E}(t) \hat{z}_{I}-i \eta \hat{W}_{I}\right)\left|\Psi_{I}(t)\right\rangle$.

Because of the CAP, the norm $\left\|\Psi_{I}(t)\right\|^{2}$ is not conserved. That affects the time evolution of the trace of the IDM

$$
\begin{aligned}
\frac{\partial}{\partial t} \operatorname{Tr}_{i}\left[\hat{\tilde{\rho}}_{I}^{\mathrm{IDM}}(t)\right]+\frac{\partial}{\partial t}\left|\alpha_{0}(t)\right|^{2} & =\frac{\partial}{\partial t}\left\langle\Psi_{I}(t) \mid \Psi_{I}(t)\right\rangle \\
& =-2 \eta \operatorname{Tr}\left[\hat{W}_{I} \hat{\tilde{\rho}}_{I}(t)\right],
\end{aligned}
$$

where $\hat{\tilde{\rho}}_{I}(t)=\left|\Psi_{I}(t)\right\rangle\left\langle\Psi_{I}(t)\right|$. Since the probability that there is no hole $\left(\left|\alpha_{0}(t)\right|^{2}\right)$ and the probability that there is a hole $\left(\operatorname{Tr}_{i}\left[\hat{\tilde{\rho}}_{I}^{\mathrm{IDM}}(t)\right]\right)$ should add up to one, we must correct $\hat{\tilde{\rho}}_{I}^{\mathrm{IDM}}(t)$ for the loss of norm. 
In order to understand how to construct a corrected IDM, $\hat{\rho}_{I}^{\mathrm{IDM}}(t)$, that does not lose norm over time and describes the full interaction dynamics of the electrons, we have to analyze the equations of motion (von Neumann equations) of $\hat{\tilde{\rho}}_{I}(t)$ and $\hat{\tilde{\rho}}_{I}^{\mathrm{IDM}}(t)$

$$
\frac{\partial}{\partial t} \hat{\tilde{\rho}}_{I}(t)=-i\left[\hat{V}_{C, I}-\hat{V}_{\mathrm{HF}, I}-\mathcal{E}(t) \hat{z}_{I}, \hat{\tilde{\rho}}_{I}(t)\right]-\eta\left\{\hat{\tilde{\rho}}_{I}(t), \hat{W}_{I}\right\},
$$

$$
\begin{aligned}
\frac{\partial}{\partial t} \hat{\tilde{\rho}}_{I}^{\mathrm{IDM}}(t)= & \left.-i \operatorname{Tr}_{a} \mathbf{I}\left[\hat{V}_{C, I}-\hat{V}_{\mathrm{HF}, I}-\mathcal{E}(t) \hat{z}_{I}, \hat{\tilde{\rho}}_{I}(t)\right]\right] \\
& -2 \eta \operatorname{Tr}_{a}\left[\hat{W}_{I} \hat{\tilde{\rho}}_{I}(t)\right],
\end{aligned}
$$

where $\{\cdot, \cdot\}$ stands for the anticommutator. Equation (21a) can be derived by taking the time derivative of Eq. (14b) and using Eq. (19). In addition, we use the fact that

$$
\begin{gathered}
\operatorname{Tr}\left[\hat{W}_{I} \hat{\tilde{\rho}}_{I}(t)\right]=\operatorname{Tr}\left[\hat{\tilde{\rho}}_{I}(t) \hat{W}_{I}\right], \\
\operatorname{Tr}\left[\hat{W}_{I}\right]=\operatorname{Tr}_{a}\left[\hat{W}_{I}\right] .
\end{gathered}
$$

Remember that $\hat{W}$ acts only on virtual orbitals. The first terms on the right-hand side of Eqs. (21) describe the dynamics of the system (imaginary prefactor), and the second terms are responsible for the norm decay of the density matrix (real prefactor).

In the case of no residual Coulomb interaction $\left(\hat{V}_{C, I}-\right.$ $\left.\hat{V}_{\mathrm{HF}, I}=0\right)$ and no electric field $[\mathcal{E}(t)=0]$, the correct density matrix has to be constant in time. The density matrix $\hat{\tilde{\rho}}_{I}(t)$ does not fulfill this requirement due to the CAP. Hence, the equation of motion in Eq. (21a) has to be corrected such that no term violates the norm conservation. The corresponding von Neumann equations of the corrected density matrix and the corrected IDM are

$$
\begin{aligned}
\frac{\partial}{\partial t} \hat{\rho}_{I}(t) & =\frac{\partial}{\partial t} \hat{\tilde{\rho}}_{I}(t)+\eta\left\{\hat{\tilde{\rho}}_{I}(t), \hat{W}_{I}\right\} \\
& =-i\left[\hat{V}_{C, I}-\hat{V}_{\mathrm{HF}, I}-\mathcal{E}(t) \hat{z}_{I}, \hat{\tilde{\rho}}_{I}(t)\right], \\
\frac{\partial}{\partial t} \hat{\rho}_{I}^{\mathrm{IDM}}(t) & =\frac{\partial}{\partial t} \hat{\tilde{\rho}}_{I}^{\mathrm{IDM}}(t)+2 \eta \operatorname{Tr}_{a}\left[\hat{W}_{I} \hat{\tilde{\rho}}_{I}(t)\right] \\
& \left.=-i \operatorname{Tr}_{a} \mathbf{I}\left[\hat{V}_{C, I}-\hat{V}_{\mathrm{HF}, I}-\mathcal{E}(t) \hat{z}_{I}, \hat{\tilde{\rho}}_{I}(t)\right]\right] .
\end{aligned}
$$

The corrected density matrices, as defined in Eqs. (23), experience no damping. The norm of $\hat{\rho}_{I}(t)$ is conserved, which can be seen by taking the trace of Eq. (23a). By just taking the trace over all $1 \mathrm{p}-1 \mathrm{~h}$ excitations, Eq. (23a) reduces to

$$
\begin{aligned}
\frac{\partial}{\partial t}\left(\operatorname{Tr}_{i}\left[\hat{\rho}_{I}^{\mathrm{IDM}}(t)\right]\right) & \left.=-i \operatorname{Tr}_{i} \operatorname{Tr}_{a} \mathrm{I}\left[\hat{V}_{C, I}-\hat{V}_{\mathrm{HF}, I}-\mathcal{E}(t) \hat{z}_{I}, \hat{\tilde{\rho}}_{I}(t)\right]\right] \\
& =-i\left\langle\Phi_{0}\left|\left[\mathcal{E}(t) \hat{z}_{I}, \hat{\tilde{\rho}}^{I}(t)\right]\right| \Phi_{0}\right\rangle \\
& =-\frac{\partial}{\partial t}\left|\alpha_{0}(t)\right|^{2} .
\end{aligned}
$$

In the second step in Eq. (24), we used

$$
\begin{aligned}
\left(\Phi_{i}^{a}\left|\hat{V}_{C, I}-\hat{V}_{\mathrm{HF}, I}\right| \Phi_{0}\right) & =\left(\Phi_{i}^{a}\left|\hat{z}_{I}\right| \Phi_{i}^{a}\right) \\
& =\left(\Phi_{0}\left|\hat{z}_{I}\right| \Phi_{0}\right)=0, \quad \forall a, i .
\end{aligned}
$$

Hence, we can conclude that after the pulse $\mathcal{E}(t)=0$, where $\frac{\partial}{\partial t}\left|\alpha_{0}(t)\right|^{2}=0$, the trace of $\hat{\rho}_{I}^{\mathrm{IDM}}(t)$ is constant in time. In addition, $\hat{\rho}_{I}(t)$ fulfills the requirement [see Eq. (24)] that the probability of leaving the atom in its ground state $\left(\left|\alpha_{0}(t)\right|^{2}\right)$ and the probability to create $1 \mathrm{p}-1 \mathrm{~h}$ excitations $\left(\operatorname{Tr}_{i}\left[\hat{\rho}_{I}^{\mathrm{IDM}}(t)\right]\right)$ add up to one at all times.

Since $\hat{\tilde{\rho}}^{\mathrm{IDM}}(t)$ can be calculated quite efficiently from the solutions of Eqs. (5a) and (5b), it is sufficient to calculate the correction matrix

$$
\delta \hat{\rho}_{I}^{\mathrm{IDM}}(t)=\hat{\rho}_{I}^{\mathrm{IDM}}(t)-\hat{\tilde{\rho}}_{I}^{\mathrm{IDM}}(t) .
$$

The equation of motion of $\delta \hat{\rho}_{I}^{\mathrm{IDM}}(t)$ can be derived from Eqs. (23b) and (26),

$$
\begin{aligned}
\frac{\partial}{\partial t} \delta \hat{\rho}_{I}^{\mathrm{IDM}}(t) & =2 \eta \operatorname{Tr}_{a}\left[\hat{W}_{I} \hat{\tilde{\rho}}_{I}(t)\right] \\
& =-i \operatorname{Tr}_{a} \mathbf{I}\left[-i \eta \hat{W}_{I}, \delta \hat{\rho}_{I}(t)\right] \mathbf{]}+2 \eta \operatorname{Tr}_{a}\left[\hat{W}_{I} \hat{\tilde{\rho}}_{I}(t)\right]
\end{aligned}
$$

where we used Eqs. (22) to insert a commutator that includes the CAP. In the Schrödinger picture, Eq. (27) transforms into

$$
\frac{\partial}{\partial t} \delta \rho_{i, j}^{\mathrm{IDM}}(t)=i\left(\varepsilon_{i}-\varepsilon_{j}\right) \delta \rho_{i, j}^{\mathrm{IDM}}(t)+2 \eta \sum_{a, b} w_{b, a} \alpha_{i}^{a}(t)\left[\alpha_{j}^{b}(t)\right]^{*} .
$$

Here, it was exploited that $\sum_{c} o_{b, c} w_{(c, a)}=\left\langle\varphi_{b}|\hat{W}| \varphi_{a}\right\rangle=w_{b, a}$. The solution to Eq. (28) is

$$
\begin{aligned}
\delta \rho_{i, j}^{\mathrm{IDM}}(t)= & 2 \eta e^{i\left(\varepsilon_{i}-\varepsilon_{j}\right) t} \int_{-\infty}^{t} d t^{\prime} \sum_{a, b} w_{b, a} \alpha_{i}^{a}\left(t^{\prime}\right) \\
& \times\left[\alpha_{j}^{b}\left(t^{\prime}\right)\right]^{*} e^{-i\left(\varepsilon_{i}-\varepsilon_{j}\right) t^{\prime}}
\end{aligned}
$$

and hence, we have found an expression for the corrected IDM,

$$
\begin{aligned}
\rho_{i, j}^{\mathrm{IDM}}(t)= & \tilde{\rho}_{i, j}^{\mathrm{IDM}}(t)+2 \eta e^{i\left(\varepsilon_{i}-\varepsilon_{j}\right) t} \int_{-\infty}^{t} d t^{\prime} \\
& \times \sum_{a, b} w_{b, a} \alpha_{i}^{a}\left(t^{\prime}\right)\left[\alpha_{j}^{b}\left(t^{\prime}\right)\right]^{*} e^{-i\left(\varepsilon_{i}-\varepsilon_{j}\right) t^{\prime}} .
\end{aligned}
$$

Equation (30) is identical to the definition of the IDM in Ref. [89], where the states $\left|\Phi_{i}^{a}\right\rangle$ are eigenstates of $\hat{F}$ rather than $\hat{F}_{\text {CAP }}$ and fulfill different orthogonality relations. Therefore, it was not obvious that both sets of basis states lead to a formally identical expression. In contrast to Ref. [89], the exact Coulomb interaction is considered in the current treatment. As a consequence of the Coulomb interaction between the excited electron and the ion, the IDM in the interaction picture is not necessarily constant after the laser pulse is over. However, since within the one-hole configuration space, the one-hole channel states are eigenstates of the exact, laser-free Hamiltonian, the IDM in the interaction picture becomes constant if the excited electron is unbound and drifts away from the ion.

\section{E. Atomic Hartree-Fock equations}

The theory formulated in Secs. II A-II D holds for any electronic system where the Born-Oppenheimer approximation can be made. In the following, we focus our discussion on closed-shell atoms, where the total orbital and spin angular 
momenta of the ground state are 0 . The Fock operator for a closed-shell atom is given by [110]

$$
\hat{F}=-\frac{1}{2} \nabla^{2}-\frac{Z}{r}+\sum_{i}\left(2 J_{i}-K_{i}\right),
$$

which consists of a kinetic part $\left(-\nabla^{2} / 2\right)$, a Coulomb potential due to the nucleus $(-Z / r)$, and direct $\left(2 J_{i}\right)$ and exchange $\left(K_{i}\right)$ Coulomb interactions between the electrons. The CAP used in this work (see Sec. II B) preserves the spherical symmetry of the atomic-structure problem, so that the one-electron eigenfunctions of $\hat{F}_{\mathrm{CAP}}=\hat{F}-i \eta \hat{W}$ may be written as a product of a (generally complex) radial function $u_{n, l}$ and a spherical harmonic $Y_{l, m}(\theta, \phi)$,

$$
\left.\left\langle r, \theta, \phi \mid \varphi_{p}\right\rangle=\langle r, \theta, \phi| \varphi_{p}\right)=\varphi_{p}(r, \theta, \phi)=\frac{u_{n, l}(r)}{r} Y_{l, m}(\theta, \phi) .
$$

The $u_{n, l}$ satisfy the radial self-consistent-field equations,

$$
\begin{aligned}
- & \frac{1}{2} \frac{d^{2} u_{n, l}(r)}{d r^{2}}+\left\{\frac{l(l+1)}{2 r^{2}}-\frac{Z}{r}-i \eta W(r)\right\} u_{n, l}(r) \\
& +\sum_{n_{o}, l_{o}}\left(4 l_{o}+2\right) v^{(0)}\left(n_{o}, l_{o} ; n_{o}, l_{o} ; r\right) u_{n, l}(r) \\
& -\sum_{n_{o}, l_{o}} \sum_{L=\left|l-l_{o}\right|}^{l+l_{o}} C\left(l, L, l_{o} ; 0,0,0\right)^{2} v^{(L)}\left(n_{o}, l_{o} ; n, l ; r\right) u_{n_{o}, l_{o}}(r) \\
= & \varepsilon_{n, l} u_{n, l}(r),
\end{aligned}
$$

where

$$
\begin{gathered}
v^{(L)}\left(n, l ; n^{\prime}, l^{\prime} ; r_{1}\right)=\int_{0}^{\infty} d r_{2} \frac{r_{<}^{L}}{r_{>}^{L+1}} u_{n, l}\left(r_{2}\right) u_{n^{\prime}, l^{\prime}}\left(r_{2}\right), \\
r_{<}=\min \left\{r_{1}, r_{2}\right\}, \quad r_{>}=\max \left\{r_{1}, r_{2}\right\},
\end{gathered}
$$

and $C\left(l_{1}, l_{2}, l_{3} ; m_{1}, m_{2}, m_{3}\right)$ is a Clebsch-Gordan coefficient [111]. The summation over $n_{o}, l_{o}$ in Eq. (33) extends over all subshells occupied in the Hartree-Fock ground state. In our calculations, the $u_{n, l}(r)$ are subject to the boundary conditions $u_{n, l}(0)=0$ and $u_{n, l}\left(r_{\max }\right)=0$. Here, $r_{\max }$ stands for the end point of the numerical grid employed (see Sec. III).

The Hartree-Fock mean-field potential, $\sum_{i}\left(2 J_{i}-K_{i}\right)$, depends only on the occupied orbitals. However, all orbitalsoccupied and virtual - are influenced by the mean-field potential. On the other hand, since the CAP we have introduced in Sec. II B starts far away from the origin such that occupied orbitals are not affected, the Hartree-Fock mean field does not depend on the CAP. Therefore, the occupied orbitals $\varphi_{i}$ are eigenstates of both $\hat{F}$ and $\hat{F}_{\mathrm{CAP}}$, and may be calculated by solving the self-consistent-field problem, Eq. (33), assuming $\eta=0$. After determining the $\varphi_{i}$, and thus fixing the HartreeFock mean field, a single diagonalization of $\hat{F}_{\text {CAP }}$ for nonzero $\eta$ gives the virtual orbitals $\varphi_{a}$.

The dual wave function of $\left|\varphi_{p}\right\rangle$ is $\left(\varphi_{p} \mid\right.$ rather than $\left\langle\varphi_{p}\right|$, as discussed in Sec. II C. The matrix representation of Eq. (33) in a real basis gives rise to a complex symmetric eigenvalue problem [106] (see Sec. III B). From this and from Eq. (32), it follows that the spatial representation of $\left(\varphi_{p} \mid\right.$ is given by

$$
\left(\varphi_{p}|r, \theta, \phi\rangle=Y_{l_{p}, m_{p}}^{*}(\theta, \phi) \frac{u_{n_{p}, l_{p}}(r)}{r} .\right.
$$

The corresponding orthogonality relations were given in Sec. II C.

\section{NUMERICAL IMPLEMENTATION}

In this section, we present our numerical method to implement the three-dimensional TDCIS. In Sec. III A, our choice of the pseudospectral grid for the radial degree of freedom is discussed. Then, the atomic Hartree-Fock equations on the numerical grid are shown in Sec. III B. In Secs. III D and III E we describe propagation schemes based on two different ways of partitioning the Hamiltonian in Eq. (4).

\section{A. Pseudospectral grid}

Due to the nature of the strong-field problem, a grid is required with sufficient density near the atomic nucleus to describe the electronic structure of the atom, as well as far from the nucleus to describe the wave packet of the ejected electron. We have chosen a Gauss-Lobatto grid [112-115], which uses as its grid points the roots of the first derivative of the $N$ th-order Legendre polynomial $\left(P_{N}^{\prime}\right)$ as well as the selected end points of the grid $\left(r=0\right.$ and $\left.r=r_{\max }\right)$. We map these roots, which lie on the interval $x \in[-1,1]$, onto the radial space of the atom [113] using

$$
r(x)=L \frac{1+x}{1-x+\zeta} .
$$

$L$ and $\zeta$ are parameters which control the extent of the grid and the density of the mapped points near the origin, respectively.

As described in Ref. [113], a wave function $\phi(x)$ may be approximated using a finite basis set of orthogonal polynomials. Using Legendre polynomials $P_{l}(x)$,

$$
\phi(x) \approx \phi_{N}(x)=\sum_{l=0}^{N} a_{l} P_{l}(x),
$$

which may also be written in terms of cardinal functions $g_{k}(x)$,

$$
\phi_{N}(x)=\sum_{k=0}^{N} g_{k}(x) \phi_{N}\left(x_{k}\right) .
$$

Analytical functions of the wave function $\phi(x)$ can now be written in terms of analytical functions of the cardinal functions. For the Gauss-Lobatto grid points, the second derivative of $g_{k}(x)$, which is needed to calculate the radial kinetic energy, is (for grid points not at the edge of the grid, which we will not need)

$$
g_{k}^{\prime \prime}\left(x_{k^{\prime}}\right)=d_{k, k^{\prime}}^{(2)} \frac{P_{N}\left(x_{k^{\prime}}\right)}{P_{N}\left(x_{k}\right)},
$$

where

$$
\begin{aligned}
d_{k, k}^{(2)} & =-\frac{N(N+1)}{3\left(1-x_{k}^{2}\right)}, \\
d_{k, k^{\prime}}^{(2)} & =-\frac{2}{\left(x_{k}-x_{k^{\prime}}\right)^{2}} .
\end{aligned}
$$




\section{B. Atomic Hartree-Fock equations in the Gauss-Lobatto grid}

After employing the mapping of Eq. (37), the Hartree-Fock equations [Eq. (31)] read

$$
\begin{aligned}
-\frac{1}{2}( & \left.-\frac{r^{\prime \prime}(x)}{r^{\prime}(x)^{3}} \frac{d}{d x}+\frac{1}{r^{\prime}(x)^{2}} \frac{d^{2}}{d x^{2}}\right) u_{n, l}(x) \\
& +\left\{\frac{l(l+1)}{2 r(x)^{2}}-\frac{Z}{r(x)}-i \eta W(r(x))\right\} u_{n, l}(x) \\
& +\sum_{n_{o}, l_{o}}\left(4 l_{o}+2\right) v^{(0)}\left(n_{o}, l_{o} ; n_{o}, l_{o} ; r(x)\right) u_{n, l}(x) \\
& -\sum_{n_{o}, l_{o}} \sum_{L=\left|l-l_{o}\right|}^{l+l_{o}} C\left(l L l_{o} ; 000\right)^{2} v^{(L)}\left(n_{o}, l_{o} ; n, l ; r(x)\right) u_{n_{o}, l_{o}}(x) \\
= & \varepsilon_{n, l} u_{n, l}(x) .
\end{aligned}
$$

In order to symmetrize the Laplacian, the function $A(x)$ is introduced such that

$$
A_{n, l}(x)=\sqrt{r^{\prime}(x)} u_{n, l}(x) .
$$

After expanding $A(x)$ with Eq. (39), substituting the relation $g_{k}\left(x_{k^{\prime}}\right)=\delta_{k, k^{\prime}}$ and $A_{n, l}^{k}=A_{n, l}\left(x_{k}\right) / P_{N}\left(x_{k}\right)$ and using Eqs. (40) and (41), we obtain

$$
\begin{aligned}
\sum_{k}\{ & \left.-\frac{1}{2} \frac{1}{r^{\prime}\left(x_{k^{\prime}}\right)} d_{k, k^{\prime}}^{(2)} \frac{1}{r^{\prime}\left(x_{k^{\prime}}\right)} A_{n, l}^{k}\right\} \\
& +\left\{\frac{l(l+1)}{2 r\left(x_{k^{\prime}}\right)^{2}}-\frac{Z}{r\left(x_{k^{\prime}}\right)}-i \eta W\left(r\left(x_{k^{\prime}}\right)\right)\right\} A_{n, l}^{k^{\prime}} \\
& +\sum_{n_{o}, l_{o}}\left(4 l_{o}+2\right) v^{(0)}\left(n_{o}, l_{o} ; n_{o}, l_{o} ; r\left(x_{k^{\prime}}\right)\right) A_{n, l}^{k^{\prime}} \\
& -\sum_{n_{o}, l_{o}} \sum_{L=\left|l-l_{o}\right|}^{l+l_{o}} C\left(l L l_{o} ; 000\right)^{2} v^{(L)}\left(n_{o}, l_{o} ; n, l ; r\left(x_{k^{\prime}}\right)\right) A_{n_{o}, l_{o}}^{k^{\prime}} \\
= & \varepsilon_{n, l} A_{n, l}^{k^{\prime}} .
\end{aligned}
$$

The integrals of Eq. (34) can now be written using the quadrature

$$
v^{(L)}\left(n, l ; n^{\prime}, l^{\prime} ; r\left(x_{k^{\prime}}\right)\right)=\sum_{k} \frac{r\left(k, k^{\prime}\right)_{<}^{L}}{r\left(k, k^{\prime}\right)_{>}^{L+1}} A_{n, l}^{k} A_{n^{\prime}, l^{\prime}}^{k} \frac{2}{N(N+1)},
$$

where

$$
\begin{aligned}
& r\left(k, k^{\prime}\right)_{<}=\min \left\{r\left(x_{k}\right), r\left(x_{k^{\prime}}\right)\right\}, \\
& r\left(k, k^{\prime}\right)_{>}=\max \left\{r\left(x_{k}\right), r\left(x_{k^{\prime}}\right)\right\} .
\end{aligned}
$$

Substituting this integral into Eq. (44), the atomic HartreeFock equations can be written as

$$
\begin{aligned}
\sum_{k}\{ & \left.-\frac{1}{2} \frac{1}{r^{\prime}\left(x_{k^{\prime}}\right)} d_{k, k^{\prime}}^{(2)} \frac{1}{r^{\prime}\left(x_{k^{\prime}}\right)} A_{n, l}^{k}\right\} \\
& +\left\{\frac{l(l+1)}{2 r\left(x_{k^{\prime}}\right)^{2}}-\frac{Z}{r\left(x_{k^{\prime}}\right)}-i \eta W\left(r\left(x_{k^{\prime}}\right)\right)\right\} A_{n, l}^{k^{\prime}} \\
& +\sum_{k, n_{o}, l_{o}} \frac{2}{N(N+1)}\left(4 l_{o}+2\right)\left|A_{n_{o}, l_{o}}^{k}\right|^{2} \frac{1}{r\left(k, k^{\prime}\right)_{>}} A_{n, l}^{k^{\prime}}
\end{aligned}
$$

$$
\begin{aligned}
& -\sum_{k, n_{o}, l_{o}} \sum_{L=\left|l-l_{o}\right|}^{l+l_{o}} \frac{2}{N(N+1)} C\left(l L l_{o} ; 000\right)^{2} \\
& \times \frac{r\left(k, k^{\prime}\right)_{<}^{L}}{r\left(k, k^{\prime}\right)_{>}^{L+1}} A_{n_{o}, l_{o}}^{k^{\prime}} A_{n_{o}, l_{o}}^{k} A_{n, l}^{k}=\varepsilon_{n, l} A_{n, l}^{k^{\prime}},
\end{aligned}
$$

which is a complex symmetric eigenvalue problem.

\section{Approximation for large angular-momentum Coulomb matrix elements}

In the context of TDCIS, two types of Coulomb matrix elements of the form $v_{\left(a, i^{\prime}, i, a^{\prime}\right)}$ and $v_{\left(a, i^{\prime}, a^{\prime}, i\right)}$ are of interest. In the case that excited orbitals with large angular momenta are important, a substantial number of Coulomb matrix elements need to be computed. This computational task can be the bottleneck in solving the TDCIS equations.

To make this task manageable, we employ the approximations

$$
\begin{gathered}
v_{\left(a, i^{\prime}, i, a^{\prime}\right)}=0, \\
v_{\left(a, i^{\prime}, a^{\prime}, i\right)}=\delta_{i, i^{\prime}}\left(\varphi_{a}\left|\frac{1}{r}\right| \varphi_{a^{\prime}}\right),
\end{gathered}
$$

if either $l_{a}$ or $l_{a^{\prime}}$ is larger than a threshold value $L_{\text {coul }}$. These approximations are valid for an excited electron that is far away from the residual ion. We choose the value of $L_{\text {coul }}$ such that numerical convergence is reached.

\section{Numerical propagation scheme}

In order to propagate the ground-state coefficient $\alpha_{0}$, we directly apply the second-order finite-differencing scheme [116] to Eq. (5a):

$$
\alpha_{0}(t+d t)=\alpha_{0}(t-d t)+2 i d t \sqrt{2} \mathcal{E}(t) \sum_{i} \sum_{a} \alpha_{i}^{a}(t) z_{(i, a)} .
$$

To propagate the coefficients $\alpha_{i}^{a}(t)$, we temporarily transform to the interaction picture,

$$
\alpha_{i}^{a}(t)=e^{-i\left(\varepsilon_{a}-\varepsilon_{i}\right) t} \tilde{\alpha}_{i}^{a}(t) .
$$

The propagation equation for $\tilde{\alpha}_{i}^{a}$ becomes

$$
\dot{\tilde{\alpha}}_{i}^{a}(t)=-i e^{i\left(\varepsilon_{a}-\varepsilon_{i}\right) t} f_{i}^{a}(t),
$$

where

$$
\begin{aligned}
f_{i}^{a}(t) \equiv & \sum_{i^{\prime}} \sum_{a^{\prime}} \alpha_{i^{\prime}}^{a^{\prime}}(t)\left(2 v_{\left(a, i^{\prime}, i, a^{\prime}\right)}-v_{\left(a, i^{\prime}, a^{\prime}, i\right)}\right) \\
& -\mathcal{E}(t)\left\{\sqrt{2} \alpha_{0}(t) z_{(a, i)}+\sum_{a^{\prime}} \alpha_{i}^{a^{\prime}}(t) z_{\left(a, a^{\prime}\right)}\right. \\
& \left.-\sum_{i^{\prime}} \alpha_{i^{\prime}}^{a}(t) z_{\left(i^{\prime}, i\right)}\right\} .
\end{aligned}
$$

Now we apply the second-order differencing scheme to Eq. (51),

$$
\tilde{\alpha}_{i}^{a}(t+d t)=\tilde{\alpha}_{i}^{a}(t-d t)-2 i d t e^{i\left(\varepsilon_{a}-\varepsilon_{i}\right) t} f_{i}^{a}(t),
$$


and then convert back to the Schrödinger picture,

$$
\begin{aligned}
\alpha_{i}^{a}(t+d t) & =e^{-i\left(\varepsilon_{a}-\varepsilon_{i}\right)(t+d t)} \tilde{\alpha}_{i}^{a}(t+d t) \\
& =e^{-i\left(\varepsilon_{a}-\varepsilon_{i}\right)(t+d t)} \tilde{\alpha}_{i}^{a}(t-d t)-2 i d t e^{-i\left(\varepsilon_{a}-\varepsilon_{i}\right) d t} f_{i}^{a}(t) \\
& =e^{-2 i\left(\varepsilon_{a}-\varepsilon_{i}\right) d t} \alpha_{i}^{a}(t-d t)-2 i d t e^{-i\left(\varepsilon_{a}-\varepsilon_{i}\right) d t} f_{i}^{a}(t) .
\end{aligned}
$$

Equations (49) and (54) are the equations used to propagate the coefficients.

\section{E. Complex-orbital versus real-orbital formulation of TDCIS}

As shown in the previous section, the complex-orbital formulation of TDCIS is rather compact, because the CAP is chosen such that the occupied orbitals in the Hartree-Fock ground state remain unaffected. However, this complex-orbital formulation has the disadvantage that the usual physical interpretation of the virtual orbitals is lost. It is therefore natural to ask whether it is more advantageous to use a real-orbital formulation of TDCIS. Real radial wave functions $u_{n, l}(r)$ are obtained by diagonalizing $\hat{F}$ rather than $\hat{F}_{\mathrm{CAP}}$. The full Hamiltonian, Eq. (4), is left unmodified. Using the real-orbital formulation, all integrals and orbital energies are real. However, there is a CAP-dependent term in the equations of motion for the wave-packet expansion coefficients [which is absent in the complex-orbital formulation of Eq. (5)]. In the following, we show that even though the real-orbital and complex-orbital formulations are equivalent, the implementation of the complex-orbital formulation is numerically more stable.

For simplicity, we performed the numerical comparison using hydrogen exposed to a laser electric field with a peak amplitude of 0.1 a.u. and an angular frequency of 0.057 a.u. In this case, the $\sqrt{2}$ factors, the Coulomb matrix elements, and the dipole matrix elements $z_{i, i^{\prime}}$ in Eq. (5) disappear since there is only one electron. In these tests, 800 radial grid points, $r_{\max }=$ 130 (Sec. II E), $r_{\mathrm{abs}}=90$ (Sec. II B), $\zeta=0.4$ (Sec. III A), and $d t=0.004$ (Sec. III D) are employed. In order to accurately reflect the strong-field physics, it is important to note that the choice of CAP strength, which is characterized by $\eta$, is also important apart from these parameters. With $\eta=10^{-3}$, our calculation performed with the complex orbitals reproduces the hydrogen result obtained by Gordon and Kärtner [117]. This is not the case if a smaller value of $\eta$ is chosen. Figure 1 shows that the expectation value of the dipole acceleration obtained with $\eta=10^{-5}$ is noisier than that for $\eta=10^{-3}$. This is because the CAP with $\eta$ less than $10^{-3}$ is too weak to absorb completely the photoelectron wave packet reaching the end of the spatial grid, leading to reflections from the grid wall.

To compare the results obtained with the complex orbitals, we repeated the calculations using the real orbitals. We found that, depending on the value of $\eta$, the numerical propagation using the real-orbital formulation may not be stable. Figure 2 shows that the expectation values of the dipole acceleration obtained with the complex and real orbitals, respectively, agree with each other when $\eta=10^{-5}$. In this case, the two formulations are numerically equivalent. However, for $\eta=10^{-3}$, which is required for an accurate description of the

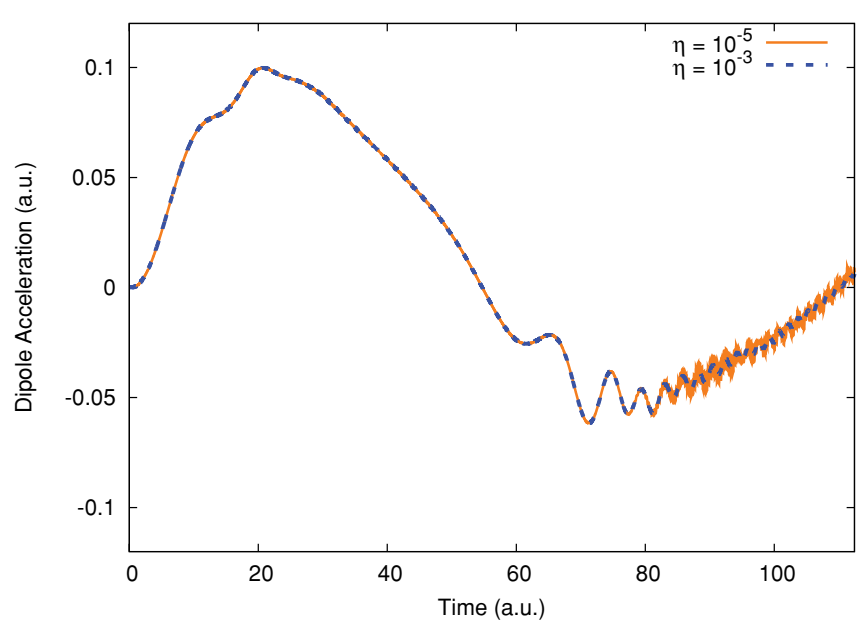

FIG. 1. (Color online) Effect of the CAP strength $\eta$ on the expectation value of the dipole acceleration calculated with the complex-orbital formulation of TDCIS.

dipole acceleration, the numerical propagation of the wave packet using the real orbitals diverges. The divergence persists even after reducing $d t$ by a factor of 10 .
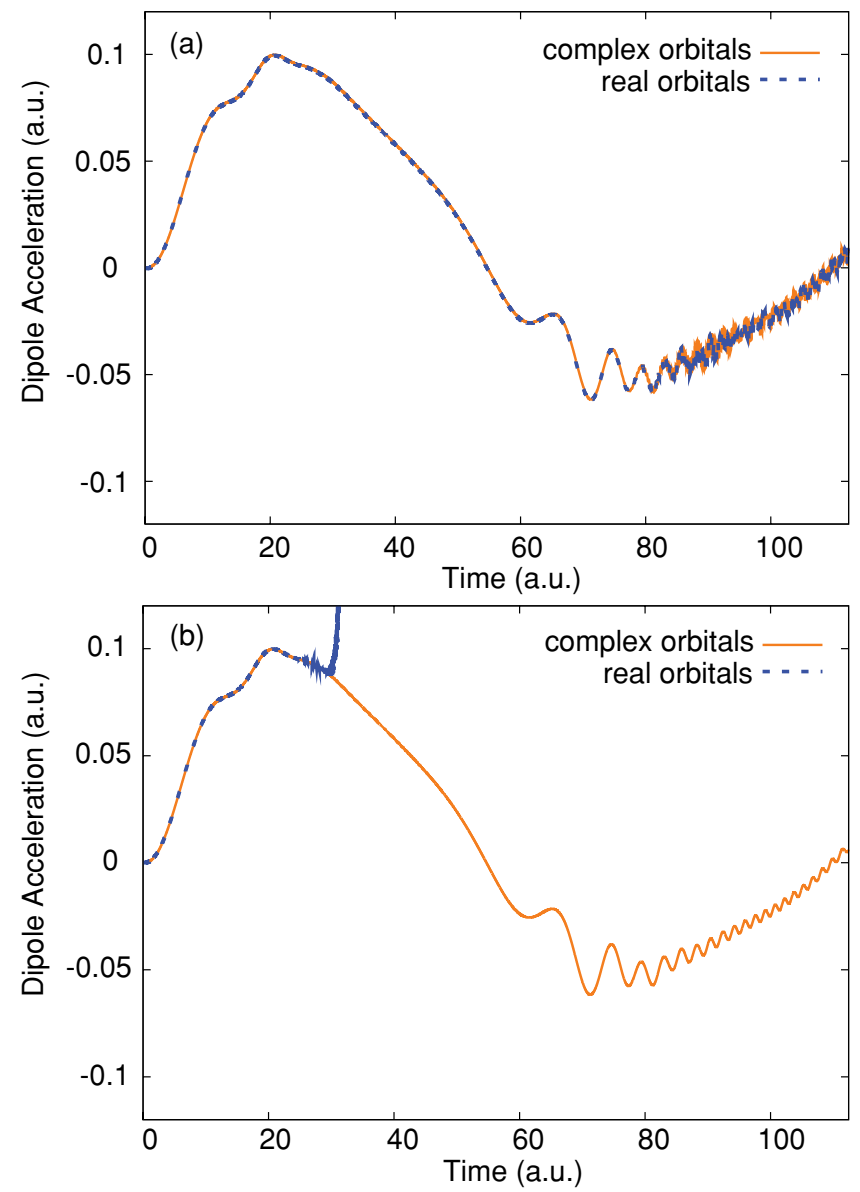

FIG. 2. (Color online) The expectation value of the dipole acceleration calculated with both the real-orbital (dashed line) and complex-orbital (solid line) formulations of TDCIS using (a) $\eta=$ $10^{-5}$ and (b) $\eta=10^{-3}$. 


\section{RESULTS AND DISCUSSION}

To demonstrate applications of our implementation of TDCIS, we performed calculations on argon. First, we carried out a convergence study with the $3 p_{0}$ orbital active and all other orbitals frozen. The laser field chosen was $\mathcal{E}(t)=$ $\mathcal{E}_{0} \sin (\omega t)$, with an $\mathcal{E}_{0}$ of 0.125 a.u. and an $\omega$ of 0.057 a.u. Classically, an electron that can recollide with its parent ion will travel $\sim \mathcal{E}_{0} / \omega^{2}=38.5$ a.u. before recollision. Therefore, to fully eliminate any CAP-induced perturbation of recolliding trajectories, we have put our absorbing potential at $r_{\mathrm{abs}}=$ 90 a.u., and our grid wall at $r_{\max }=120$ a.u., with a $\zeta$ [Eq. (37)] of 0.5 . In general, the $\zeta$ parameter was chosen as $60 / r_{\max }$.

The convergence of a number of different parameters is illustrated in Fig. 3. Figures 3(a) and 3(b) show the convergence with the CAP strength. Low $\eta$ 's produced successively greater oscillations due to reflections off the grid wall, while the large $\eta$ values did not have such obvious effects. However, close examination of large $\eta$ values such as the one shown in Fig. 3(b) showed that reflections from the CAP did affect the dipole acceleration by shifting the oscillations out toward times around 113 a.u. An $\eta$ of $5.0 \times 10^{-3}$ produced the least amount of reflections off of either the grid wall or the absorbing potential. It should be noted that this $\eta$ is in the region where the real-orbital formulation is numerically unstable. At a grid size of 500 grid points, the dependence of the dipole acceleration on the maximum angular-momentum value allowed for the dipole $\left(L_{\max }\right)$ and Coulomb $\left(L_{\text {coul }}\right)$ integrals was determined [Fig. 3(c)]. An $L_{\max }$ of 60 was determined to be necessary for convergence of the dipole acceleration, while for the Coulomb integrals an $L_{\text {coul }}$ of 4 was sufficient. For the angular-momentum quantum numbers between 5 and 60, the Coulomb interaction was approximated as a $\frac{1}{r}$ interaction (Sec. III C). Additionally, orbitals with Hartree-Fock energies higher than 50 a.u. (real part of the orbital energies) were determined to be unnecessary and were not included in the propagation calculations. Finally, the convergence of the size of the grid was found. Small grids led to extra oscillations in the dipole acceleration, as well as unphysical behaviors in the norms and density matrix elements. The argon system converged at a grid size of 750 grid points, although 1000 grid points were used for the following calculations.

Figure 4 shows the difference in the calculated dipole acceleration for the single-channel TDCIS method and the singlechannel Hartree-Slater method [39], which approximates the exchange correlation using a local function. The added effects of using the exact nonlocal exchange potential can be seen in the figure. At the peak of the dipole acceleration, the TDCIS method peaks slightly sooner than the Hartree-Slater method. The oscillation after the peak is shorter for the TDCIS method. Finally, there are a greater number of smaller oscillations after the trough of the TDCIS dipole acceleration than for the Hartree-Slater method.
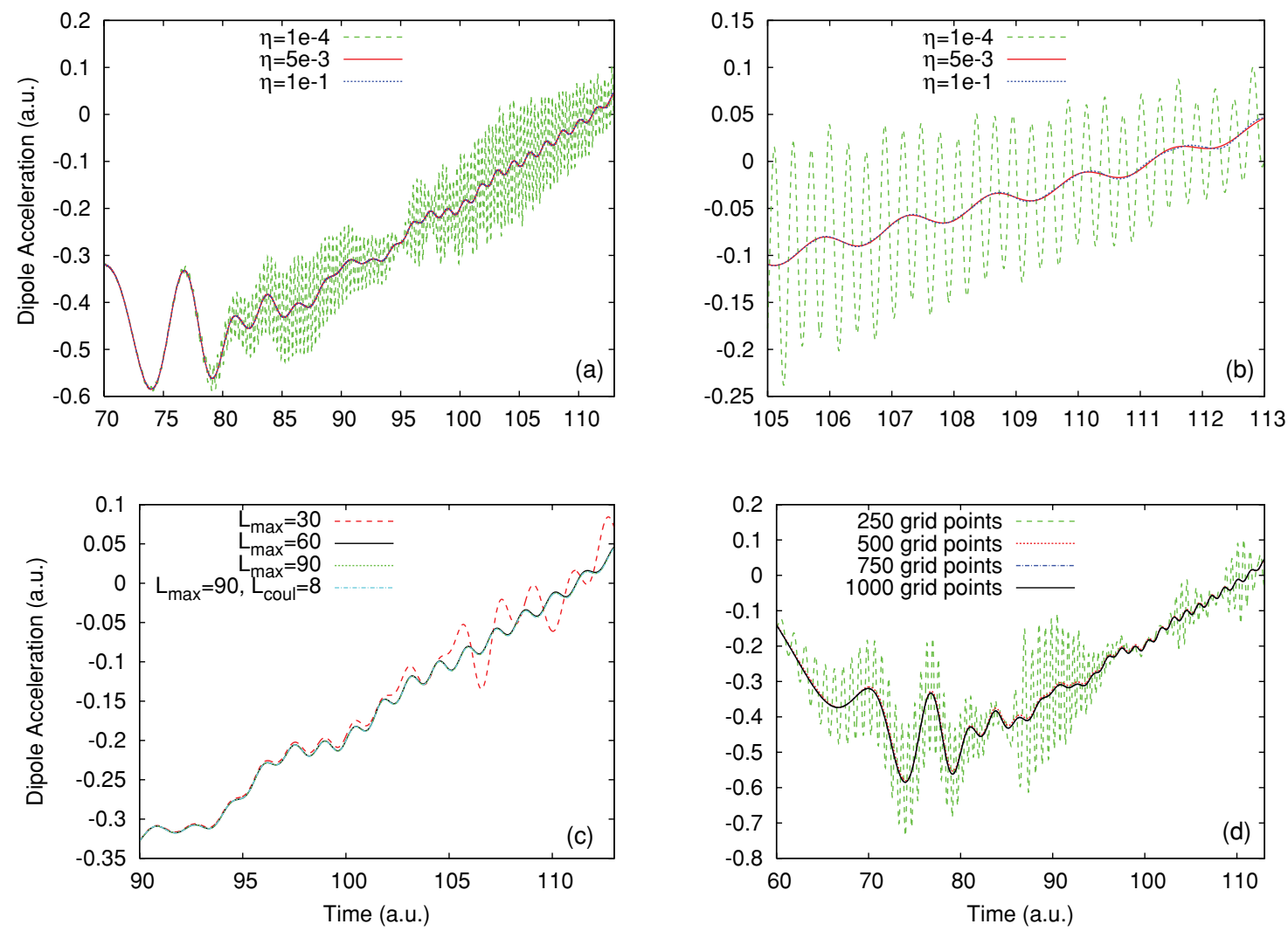

FIG. 3. (Color online) The convergence of the dipole acceleration of argon with the $3 p_{0}$ orbital active. (a), (b) Convergence with the CAP strength. (c) Convergence with maximum angular momentum for the dipole $\left(L_{\max }\right)$ and Coulomb $\left(L_{\text {coul }}\right)$ integrals. (d) Convergence with the size of the pseudospectral grid. Convergence is reached at a CAP strength of $5 \times 10^{-3}$, an $L_{\max }$ of 60 , an $L_{\text {coul }}$ of 4 , and a grid size of 750 . 


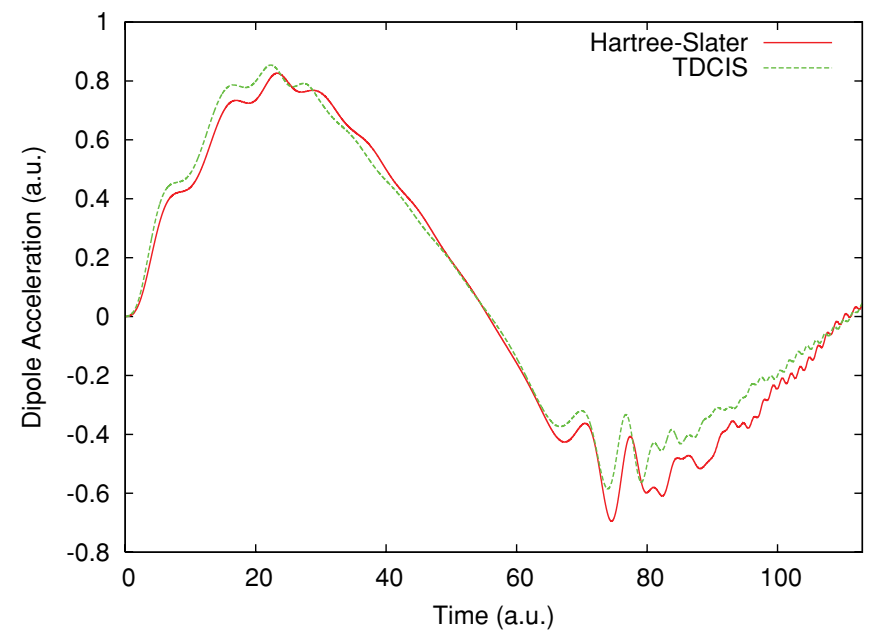

FIG. 4. (Color online) A comparison of the dipole acceleration of the single-channel TDCIS and Hartree-Slater methods. The nonlocal exchange effects are evident.

The multichannel capacity of the TDCIS framework was tested by performing calculations of the ion density matrix elements for argon with only the $3 p_{0}$ orbital active as well as with all $3 p$ orbitals active (see Fig. 5). For the case in which only the $3 p_{0}$ orbital is active, the population of the orbital increases in two steps corresponding to the two half-cycles of the driving potential. There are minor oscillations especially in the second half-cycle. When all $3 p$ orbitals are active, the population of the $3 p_{0}$ orbital follows a similar pattern but is smaller than the single-channel case. The oscillations of the $3 p_{0}$ population are slightly smaller as well for the three-channel case. Importantly, the combined populations of the $3 p_{1}$ and $3 p_{-1}$ orbitals are more than $10 \%$ of the $3 p_{0}$ population. This result conflicts with the models that ignore channel coupling and assume that these populations are sufficiently small.

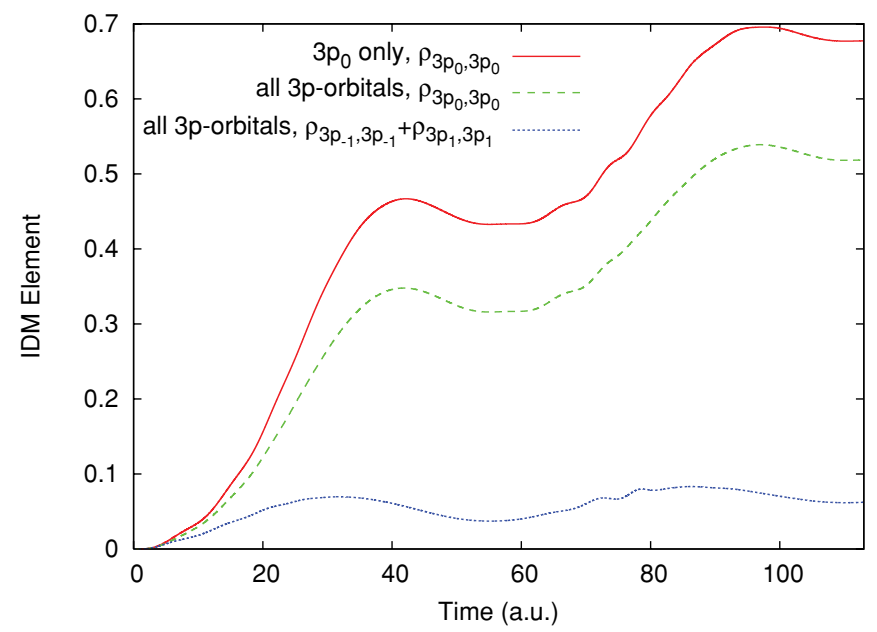

FIG. 5. (Color online) The ion density matrix elements are compared for the single-channel case and the three-channel case. The $3 p_{1}$ element plus the $3 p_{-1}$ element comprises more than $10 \%$ of the $3 p_{0}$ element in the three-channel case.

\section{CONCLUSION}

We have developed a time-dependent configurationinteraction singles formalism with a complex absorbing potential in order to describe strong-field atomic processes in the nonperturbative regime. Previous theoretical approaches have relied on a single active electron approach, while with TDCIS, multichannel processes can be investigated. Two-electron reduced density matrix (2-RDM) methods [118-122] may provide a framework in the future for examining laser-field interactions with the inclusion of explicit electron correlation and additional multireference states. The flexible pseudospectral grid allows us to calculate orbitals with sufficient density in each region of the space in order to describe weakly bound and ionized electrons as well as strongly bound atomic electrons. We have also shown that applying the CAP in the Hartree-Fock step is more numerically stable than applying it in the propagation step. This leads to a non-Hermitian modified Fock operator, as well as complex orbitals which are orthogonal with respect to a symmetric inner product. Using these complex orbitals, a reduced ion density matrix was formulated in order to determine the populations and coherences of the one-hole states. The dipole acceleration of argon was calculated as well, which requires overlap integrals between the complex orbitals.

The TDCIS dipole acceleration for argon with its $3 p_{0}$ orbital active was converged on the pseudospectral grid and then compared with the dipole acceleration calculated using the Hartree-Slater method. Local potential approximations are used in many theoretical treatments of strong-field processes. We determined that using the nonlocal exchange of the TDCIS method significantly affects the dipole acceleration. We then used the ion density matrix to compare the populations of the $3 p$ orbitals of argon in single-channel and multichannel calculations. In the single-channel calculations, only the $3 p_{0}$ orbital was allowed to be active. When all $3 p$ orbitals were open for excitation, the population of the $3 p_{0}$ orbital decreased as expected, although the time evolution was similar in shape. However, the combined $3 p_{1}$ and $3 p_{-1}$ populations were determined to be greater than $10 \%$ of the $3 p_{0}$ population. This is a significant occupation, and it shows that methods which assume a single channel do not take into account possibly important effects. The TDCIS method provides a multichannel approach for nonperturbative strong-field processes which can be used to study effects that previous theoretical methods did not take into account.

\section{ACKNOWLEDGMENTS}

We thank Nina Rohringer for helpful discussions. This work was supported by the Office of Basic Energy Sciences, US Department of Energy under Contract No. DE-AC0206CH11357. L.G. would like to thank Martha Ann and Joseph A. Chenicek and their family for financial support.

\section{APPENDIX: CALCULATING INTEGRALS}

Using the orbitals represented on a quadrature grid, we compute one- and two-body matrix elements, which are needed to solve the TDCIS equation of motion. For a general 
one-electron operator $\hat{f}$, its matrix element defined by the symmetric inner product is given by

$$
\begin{aligned}
f_{(p, q)}= & \int d r d \theta d \phi \sin \theta Y_{l_{p}, m_{p}}^{*}(\theta, \phi) u_{n_{p}, l_{p}}(r) f(r, \theta, \phi) \\
& \times Y_{l_{q}, m_{q}}(\theta, \phi) u_{n_{q}, l_{q}}(r) .
\end{aligned}
$$

We perform the quadrature, such that

$$
\begin{aligned}
f_{(p, q)}= & \frac{2}{N(N+1)} \int d \theta d \phi \sin \theta \sum_{k} A_{n_{p}, l_{p}}^{k} Y_{l_{p}, m_{p}}^{*}(\theta, \phi) \\
& \times f\left(r_{k}, \theta, \phi\right) A_{n_{q}, l_{q}}^{k} Y_{l_{q}, m_{q}}(\theta, \phi) .
\end{aligned}
$$

To obtain $f_{p, q}$, which is defined by the conjugated inner product, we replace $A_{n_{p}, l_{p}}^{k}$ in Eq. (A2) with its complex conjugate.

Using Eq. (A2), all one-electron integrals can be constructed. For the overlap integrals, the angular part becomes a $\delta$ function, and we are left with

$$
o_{p, q}=\delta_{l_{p}, l_{q}} \delta_{m_{p}, m_{q}} \sum_{k} \frac{2}{N(N+1)}\left(A_{n_{p}, l_{p}}^{k}\right)^{*} A_{n_{q}, l_{q}}^{k} .
$$

For the nonconjugated dipole integrals $(\hat{z}=r \cos \theta)$, we need for the propagation, using the relation $Y_{1,0}=\frac{4}{3 \pi} \cos \theta$ and the fact that the integral of three spherical harmonics can be written using Clebsch-Gordan coefficients [111],

$$
\begin{aligned}
& z_{(p, q)}=\sqrt{\frac{2 l_{q}+1}{2 l_{p}+1}} C\left(l_{q}, 1, l_{p} ; 0,0,0\right) C\left(l_{q}, 1, l_{p} ; m_{q}, 0, m_{p}\right) \\
& \times \sum_{k} \frac{2}{N(N+1)} A_{n_{p}, l_{p}}^{k} A_{n_{q}, l_{q}}^{k} r\left(x_{k}\right) .
\end{aligned}
$$

We also need the conjugated dipole acceleration integrals $(\hat{a}=$ $Z \cos \theta / r^{2}$ ),

$$
\begin{aligned}
a_{p, q}= & \sqrt{\frac{2 l_{q}+1}{2 l_{p}+1}} C\left(l_{q}, 1, l_{p} ; 0,0,0\right) C\left(l_{q}, 1, l_{p} ; m_{q}, 0, m_{p}\right) \\
& \times \sum_{k} \frac{2}{N(N+1)}\left(A_{n_{p}, l_{p}}^{k}\right)^{*} A_{n_{q}, l_{q}}^{k} \frac{Z}{r\left(x_{k}\right)^{2}} .
\end{aligned}
$$

The nonconjugated two-electron Coulomb integrals are also required for the propagation, and they are constructed using a two-index quadrature. The expansion

$$
\frac{1}{r_{12}}=\sum_{L=0}^{\infty} \frac{4 \pi}{2 L+1} \frac{r_{<}^{L}}{r_{>}^{L+1}} \sum_{M=-L}^{L} Y_{L, M}^{*}\left(\theta_{1}, \phi_{1}\right) Y_{L, M}\left(\theta_{2}, \phi_{2}\right)
$$

is used. Thus, the angular part of the Coulomb integrals reduce to Clebsch-Gordan coefficients, and the radial part becomes

$$
v_{(p, q, s, t)} \Rightarrow \sum_{k, k^{\prime}} A_{n_{p}, l_{p}}^{k} A_{n_{q}, l_{q}}^{k^{\prime}} A_{n_{s}, l_{s}}^{k} A_{n_{t}, l_{t}}^{k^{\prime}} \frac{r\left(k, k^{\prime}\right)_{<}^{L}}{r\left(k, k^{\prime}\right)_{>}^{L+1}} .
$$

[1] L. V. Keldysh, Zh. Eksp. Teor. Fiz. 47, 1945 (1964) [Sov. Phys. JETP 20, 1307 (1965)].

[2] A. M. Perelomov, V. S. Popov, and M. V. Terentev, Zh. Eksp. Teor. Fiz. 50, 1393 (1965) [Sov. Phys. JETP 23, 924 (1966)].

[3] F. H. M. Faisal, J. Phys. B 6, L89 (1973).

[4] L. A. Lompre, G. Mainfray, C. Manus, S. Repoux, and J. Thebault, Phys. Rev. Lett. 36, 949 (1976).

[5] H. R. Reiss, Phys. Rev. A 22, 1786 (1980).

[6] P. Lambropoulos, Phys. Rev. Lett. 55, 2141 (1985).

[7] M. V. Ammosov, N. B. Delone, and V. P. Krainov, Zh. Eksp. Teor. Fiz. 91, 2008 (1986) [Sov. Phys. JETP 64, 1191 (1986)].

[8] F. Yergeau, S. L. Chin, and P. Lavigne, J. Phys. B 20, 723 (1987).

[9] S. Augst, D. Strickland, D. D. Meyerhofer, S. L. Chin, and J. H. Eberly, Phys. Rev. Lett. 63, 2212 (1989).

[10] T. E. Glover, J. K. Crane, M. D. Perry, R. W. Lee, and R. W. Falcone, Phys. Rev. Lett. 75, 445 (1995).

[11] G. N. Gibson, M. Li, C. Guo, and J. Neira, Phys. Rev. Lett. 79, 2022 (1997).

[12] J. Muth-Böhm, A. Becker, and F. H. M. Faisal, Phys. Rev. Lett. 85, 2280 (2000).

[13] R. Taïeb, V. Véniard, and A. Maquet, Phys. Rev. Lett. 87, 053002 (2001).

[14] R. Moshammer et al., Phys. Rev. Lett. 91, 113002 (2003).

[15] E. Gubbini, U. Eichmann, M. Kalashnikov, and W. Sandner, Phys. Rev. Lett. 94, 053602 (2005).

[16] O. Smirnova, M. Spanner, and M. Ivanov, Phys. Rev. A 77, 033407 (2008).
[17] P. Eckle, A. N. Pfeiffer, C. Cirelli, A. Staudte, R. Dörner, H. G. Muller, M. Büttiker, and U. Keller, Science 322, 1525 (2008).

[18] P. Agostini, F. Fabre, G. Mainfray, G. Petite, and N. K. Rahman, Phys. Rev. Lett. 42, 1127 (1979).

[19] R. R. Freeman, P. H. Bucksbaum, H. Milchberg, S. Darack, D. Schumacher, and M. E. Geusic, Phys. Rev. Lett. 59, 1092 (1987).

[20] P. B. Corkum, N. H. Burnett, and F. Brunel, Phys. Rev. Lett. 62, 1259 (1989).

[21] E. Mevel, P. Breger, R. Trainham, G. Petite, P. Agostini, J. P. Chambaret, A. Migus, and A. Antonetti, J. Phys. B 25, L401 (1992).

[22] E. Mevel, P. Breger, R. Trainham, G. Petite, P. Agostini, A. Migus, J. P. Chambaret, and A. Antonetti, Phys. Rev. Lett. 70, 406 (1993).

[23] U. Mohideen, M. H. Sher, H. W. K. Tom, G. D. Aumiller, O. R. Wood, R. R. Freeman, J. Boker, and P. H. Bucksbaum, Phys. Rev. Lett. 71, 509 (1993).

[24] G. G. Paulus, F. Zacher, H. Walther, A. Lohr, W. Becker, and M. Kleber, Phys. Rev. Lett. 80, 484 (1998).

[25] T. Morishita, A.-T. Le, Z. Chen, and C. D. Lin, Phys. Rev. Lett. 100, 013903 (2008).

[26] M. Okunishi, T. Morishita, G. Prümper, K. Shimada, C. D. Lin, S. Watanabe, and K. Ueda, Phys. Rev. Lett. 100, 143001 (2008).

[27] M. Busuladžić, A. Gazibegović-Busuladžić, D. B. Milošević, and W. Becker, Phys. Rev. Lett. 100, 203003 (2008). 
[28] A. McPherson, G. Gibson, H. Jara, U. Johann, T. S. Luk, I. A. McIntyre, K. Boyer, and C. K. Rhodes, J. Opt. Soc. Am. B 4, 595 (1987).

[29] M. Ferray, A. L'Huillier, X. F. Li, L. A. Lompre, G. Mainfray, and C. Manus, J. Phys. B 21, L31 (1988).

[30] A. L'Huillier, K. J. Schafer, and K. C. Kulander, Phys. Rev. Lett. 66, 2200 (1991).

[31] P. B. Corkum, Phys. Rev. Lett. 71, 1994 (1993).

[32] M. Lewenstein, Ph. Balcou, M. Yu. Ivanov, A. L'Huillier, and P. B. Corkum, Phys. Rev. A 49, 2117 (1994).

[33] Ch. Spielmann, N. H. Burnett, S. Santania, R. Koppitsch, M. Schnürer, C. Kan, M. Lenzner, P. Wobrauschek, and F. Krausz, Science 278, 661 (1997).

[34] Z. Chang, A. Rundquist, H. Wang, M. M. Murnane, and H. C. Kapteyn, Phys. Rev. Lett. 79, 2967 (1997).

[35] E. A. Gibson et al., Science 302, 95 (2003).

[36] E. A. Gibson, A. Paul, N. Wagner, R. Tobey, S. Backus, I. P. Christov, M. M. Murnane, and H. C. Kapteyn, Phys. Rev. Lett. 92, 033001 (2004).

[37] J. Seres et al., Nature 433, 596 (2005).

[38] A. Gordon and F. X. Kärtner, Phys. Rev. Lett. 95, 223901 (2005).

[39] A. Gordon, F. X. Kärtner, N. Rohringer, and R. Santra, Phys. Rev. Lett. 96, 223902 (2006).

[40] J. Levesque, D. Zeidler, J. P. Marangos, P. B. Corkum, and D. M. Villeneuve, Phys. Rev. Lett. 98, 183903 (2007).

[41] M. Spanner and P. Brumer, Phys. Rev. A 78, 033840 (2008).

[42] G. Jordan and A. Scrinzi, New J. Phys. 10, 025035 (2008).

[43] B. Walker, B. Sheehy, L. F. DiMauro, P. Agostini, K. J. Schafer, and K. C. Kulander, Phys. Rev. Lett. 73, 1227 (1994).

[44] Th. Weber et al., Nature 405, 658 (2000).

[45] R. Moshammer et al., Phys. Rev. Lett. 84, 447 (2000).

[46] A. Becker and F. H. M. Faisal, Phys. Rev. Lett. 84, 3546 (2000).

[47] R. Kopold, W. Becker, H. Rottke, and W. Sandner, Phys. Rev. Lett. 85, 3781 (2000).

[48] A. S. Alnaser et al., Phys. Rev. Lett. 93, 183202 (2004).

[49] P. J. Ho, R. Panfili, S. L. Haan, and J. H. Eberly, Phys. Rev. Lett. 94, 093002 (2005).

[50] S. Gräfe and M. Yu. Ivanov, Phys. Rev. Lett. 99, 163603 (2007).

[51] P. Koval, F. Wilken, D. Bauer, and C. H. Keitel, Phys. Rev. Lett. 98, 043904 (2007).

[52] A. Rudenko, V. L. B. de Jesus, Th. Ergler, K. Zrost, B. Feuerstein, C. D. Schröter, R. Moshammer, and J. Ullrich, Phys. Rev. Lett. 99, 263003 (2007).

[53] M. Hentschel et al., Nature 414, 509 (2001).

[54] P. Agostini and L. F. DiMauro, Rep. Prog. Phys. 67, 813 (2004).

[55] R. Velotta, N. Hay, M. B. Mason, M. Castillejo, and J. P. Marangos, Phys. Rev. Lett. 87, 183901 (2001).

[56] H. Niikura, F. Légaré, R. Hasbani, A. D. Bandrauk, M. Yu. Ivanov, D. M. Villeneuve, and P. B. Corkum, Nature 417, 917 (2002).

[57] J. Itatani, J. Levesque, D. Zeidler, H. Niikura, H. Pépin, J. C. Kieffer, P. B. Corkum, and D. M. Villeneuve, Nature 432, 867 (2004).

[58] J. Itatani, D. Zeidler, J. Levesque, M. Spanner, D. M. Villeneuve, and P. B. Corkum, Phys. Rev. Lett. 94, 123902 (2005).

[59] Th. Ergler, A. Rudenko, B. Feuerstein, K. Zrost, C. D. Schröter, R. Moshammer, and J. Ullrich, Phys. Rev. Lett. 95, 093001 (2005).
[60] R. Santra and A. Gordon, Phys. Rev. Lett. 96, 073906 (2006).

[61] S. Patchkovskii, Z. Zhao, T. Brabec, and D. M. Villeneuve, Phys. Rev. Lett. 97, 123003 (2006).

[62] B. D. Esry, A. M. Sayler, P. Q. Wang, K. D. Carnes, and I. Ben Itzhak, Phys. Rev. Lett. 97, 013003 (2006).

[63] N. L. Wagner, A. Wüest, I. P. Christov, T. Popmintchev, X. Zhou, M. M. Murnane, and H. C. Kapteyn, Proc. Nat. Acad. Sci. USA 103, 13279 (2006).

[64] S. Baker, J. S. Robinson, C. A. Haworth, H. Teng, R. A. Smith, C. C. Chirila, M. Lein, J. W. G. Tisch, and J. P. Marangos, Science 312, 424 (2006).

[65] M. Lein, J. Phys. B 40, R135 (2007).

[66] O. Smirnova, A. S. Mouritzen, S. Patchkovskii, and M. Yu. Ivanov, J. Phys. B 40, F197 (2007).

[67] Z. Zhao, J. Yuan, and T. Brabec, Phys. Rev. A 76, 031404 (2007).

[68] S. Patchkovskii, Z. Zhao, T. Brabec, and D. M. Villeneuve, J. Chem. Phys. 126, 114306 (2007).

[69] B. Feuerstein, Th. Ergler, A. Rudenko, K. Zrost, C. D. Schröter, R. Moshammer, J. Ullrich, T. Niederhausen, and U. Thumm, Phys. Rev. Lett. 99, 153002 (2007).

[70] E. V. van der Zwan, C. C. Chirila, and M. Lein, Phys. Rev. A 78, 033410 (2008).

[71] Z. B. Walters, S. Tonzani, and C. H. Greene, J. Phys. Chem. A 112, 9439 (2008).

[72] W. Li, X. Zhou, R. Lock, S. Patchkovskii, A. Stolow, H. C. Kapteyn, and M. M. Murnane, Science 322, 1207 (2008).

[73] M. Drescher et al., Nature 419, 803 (2002).

[74] F. Lindner et al., Phys. Rev. Lett. 95, 040401 (2005).

[75] G. L. Yudin, A. D. Bandrauk, and P. B. Corkum, Phys. Rev. Lett. 96, 063002 (2006).

[76] T. Pfeifer, M. J. Abel, P. M. Nagel, A. Jullien, Z.-H. Loh, M. J. Bell, D. M. Neumark, and S. R. Leone, Chem. Phys. Lett. 463, 11 (2008).

[77] A. D. Bandrauk, S. Chelkowski, S. Kawai, and H. Lu, Phys. Rev. Lett. 101, 153901 (2008).

[78] K. C. Kulander, Phys. Rev. A 36, 2726 (1987).

[79] J. L. Krause, K. J. Schafer, and K. C. Kulander, Phys. Rev. Lett. 68, 3535 (1992).

[80] K. J. Schafer, B. Yang, L. F. DiMauro, and K. C. Kulander, Phys. Rev. Lett. 70, 1599 (1993).

[81] H. Rottke, J. Ludwig, and W. Sandner, J. Phys. B 29, 1479 (1996).

[82] P. Kaminski, R. Wiehle, V. Renard, A. Kazmierczak, B. Lavorel, O. Faucher, and B. Witzel, Phys. Rev. A 70, 053413 (2004).

[83] L. Young et al., Phys. Rev. Lett. 97, 083601 (2006).

[84] C. Höhr et al., Phys. Rev. A 75, 011403(R) (2007).

[85] Z.-H. Loh, M. Khalil, R. E. Correa, R. Santra, C. Buth, and S. R. Leone, Phys. Rev. Lett. 98, 143601 (2007).

[86] S. H. Southworth et al., Phys. Rev. A 76, 043421 (2007).

[87] N. Rohringer, A. Gordon, and R. Santra, Phys. Rev. A 74, 043420 (2006).

[88] R. Santra, R. W. Dunford, and L. Young, Phys. Rev. A 74, 043403 (2006).

[89] N. Rohringer and R. Santra, Phys. Rev. A 79, 053402 (2009).

[90] M. Lein, N. Hay, R. Velotta, J. P. Marangos, and P. L. Knight, Phys. Rev. Lett. 88, 183903 (2002).

[91] J. Zanghellini, M. Kitzler, T. Brabec, and A. Scrinzi, J. Phys. B 37, 763 (2004). 
[92] M. Nest, T. Klamroth, and P. Saalfrank, J. Chem. Phys. 122, 124102 (2005).

[93] X.-M. Tong and Shih I Chu, Phys. Rev. A 57, 452 (1998).

[94] Xi Chu and Shih I Chu, Phys. Rev. A 63, 023411 (2001).

[95] M. S. Pindzola, D. C. Griffin, and C. Bottcher, Phys. Rev. Lett. 66, 2305 (1991).

[96] K. C. Kulander, K. J. Schafer, and J. L. Krause, in Atoms in Intense Radiation Fields, edited by M. Gavrila (Academic, New York, 1992).

[97] N. E. Dahlen and R. van Leeuwen, Phys. Rev. A 64, 023405 (2001).

[98] V. Véniard, R. Taïeb, and A. Maquet, Laser Phys. 13, 465 (2003).

[99] N. Rohringer, S. Peter, and J. Burgdörfer, Phys. Rev. A 74, 042512 (2006).

[100] M. Spanner and S. Patchkovskii, Phys. Rev. A 80, 063411 (2009).

[101] N. Moiseyev, Phys. Rep. 302, 212 (1998).

[102] A. Goldberg and B. W. Shore, J. Phys. B 11, 3339 (1978).

[103] C. Leforestier and R. E. Wyatt, J. Chem. Phys. 78, 2334 (1983).

[104] G. Jolicard and E. J. Austin, Chem. Phys. Lett. 121, 106 (1985).

[105] U. V. Riss and H.-D. Meyer, J. Phys. B 26, 4503 (1993).

[106] R. Santra and L. S. Cederbaum, Phys. Rep. 368, 1 (2002).

[107] J. G. Muga, J. P. Palao, B. Navarro, and I. L. Egusquiza, Phys. Rep. 395, 357 (2004).
[108] H. A. Bethe and E. E. Salpeter, Quantum Mechanics of Oneand Two-Electron Atoms (Dover, Mineola, New York, 2008).

[109] K. Blum, Density Matrix Theory and Applications (Plenum, New York, 1996).

[110] W. R. Johnson, Atomic Structure Theory (Springer, Berlin, 2007).

[111] M. E. Rose, Elementary Theory of Angular Momentum (Dover, New York, 1995).

[112] J. P. Boyd, Chebyshev and Fourier Spectral Methods, 2nd ed. (Dover, New York, 2001).

[113] J. Wang, Shih I Chu, and C. Laughlin, Phys. Rev. A 50, 3208 (1994).

[114] G. H. Yao and Shih I Chu, Chem. Phys. Lett. 204, 381 (1993).

[115] V. Szalay, J. Chem. Phys. 99, 1978 (1993).

[116] C. Leforestier et al., J. Comput. Phys. 94, 59 (1991).

[117] A. Gordon and F. X. Kärtner, Opt. Express 13, 2941 (2005).

[118] Two-Electron Reduced-Density-Matrix Mechanics, edited by D. A. Mazziotti, Advances in Chemical Physics Vol. 134 (Wiley, New York, 2007).

[119] D. A. Mazziotti, Phys. Rev. Lett. 97, 143002 (2006).

[120] D. A. Mazziotti, Phys. Rev. A 76, 052502 (2007).

[121] G. Gidofalvi and D. A. Mazziotti, Phys. Rev. A 80, 022507 (2009).

[122] B. Schäfer-Bung and M. Nest, Phys. Rev. A 78, 012512 (2008). 